\title{
COLLECTIVE CLUSTERING DYNAMICS OF SARS-Cov-2 PARTICLES
}

Arturo Tozzi

Center for Nonlinear Science, Department of Physics, University of North Texas, Denton, Texas, USA tozziarturo@libero.it

Arturo.Tozzi@unt.edu

James F. Peters

Department of Electrical and Computer Engineering, University of Manitoba

75A Chancellor's Circle, Winnipeg, MB R3T 5V6, Canada and

Department of Mathematics, Adiyaman University, 02040 Adiyaman, Turkey,Department of Mathematics, Faculty of Arts and Sciences, Adryaman University

02040 Adiyaman, Turkey and Computational Intelligence Laboratory, University of Manitoba, WPG, MB, R3T 5V6, Canada

james.peters3@umanitoba.ca

Isabella Annesi-Maesano

French NIH (INSERM), EPAR Department, IPLESP, INSERM

Sorbonne University, Paris, France.

isabella.annesi-maesano@inserm.fr

Gennaro D'Amato

Division of Respiratory and Allergic Diseases, Department of Chest Diseases, High Specialty A. Cardarelli Hospital, Napoli, Italy

Medical School of Specialization in Respiratory Diseases, University on Naples Federico II.

gdamatomail@gmail.com

Collective spread of aggregated viral particles may have beneficial effects on viral capability to survive in the external environment, to counteract immune responses, and to successfully colonize host cells. Here we ask whether SARSCov-2 particles, responsible for COVID-19, display collective clustering behavior. Looking at microphotographs and movies of SARS-Cov-2 particles emerging from the surface of cultured cells, we describe single virions that tend to aggregate in progressively larger globular assemblies, until a network-like appearance is achieved. When SARS-Cov-2 particles stick into each other, the squeezing of single virions leads to improved viral package in host's fluids. We discuss how these findings might explain both the ability to spread of SARS-Cov-2 and the clinical severity of COVID19 in humans, paving the way to novel therapeutic strategies to mechanically disrupt collective clustering.

KEYWORDS: COVID-19; SARS-Cov; supernatant; sphere packing; electron microscopy; clustering.

Viruses may spread not just as single particles, but also as collective aggregates composed either of multiple naked virions, or virion-containing protein structures, polyploid capsids, extracellular lipid vesicles (Segredo-Otero and Sanjuán, 2019). For example, HTLV-1-infected T lymphocytes transiently store viral particles as carbohydrate-rich extracellular assemblies that recall bacterial biofilms (Pais-Correia et al., 2010; Vidakovic et al., 2018). These assemblies, held together and attached to cell surfaces by virally-induced extracellular matrix components and cellular linker proteins, allow virus spread from cell to cell (see also: Millen et al., 2019). Thoulouze and Alcover (2011) proposed that these structures at the surface of T cells from HTLV-1-infected patients, reminiscent of bacterial biofilms, could stand for "viral communities" with enhanced infectious capacity and improved spread compared with "free" viral particles.

Viral aggregates have been termed "collective infectious units" (CIU), because they might give rise to social-like virusvirus interactions that contribute to increase infection capabilities and reservoirs (Andreu-Moreno and Sanjuán, 2020). Although genetic complementation in CIU does not seem to improve viral fitness and diversity (Segredo-Otero and Sanjuán, 2019), cooperative interactions might produce favorable outcomes, such as collective dissemination, immunity evasion, labor division, and joint delivery of multiple viral genome copies to target cells which favor co-infections (Sanjuán 2017). Indeed, increasing the cellular multiplicity of infection may provide short-term benefits to viruses, such as overwhelming antiviral responses, avoiding stochastic loss of viral components, or complementing the genetic defects detectable in different genome copies (Sanjuán and Thoulouze, 2019). Virion-virion binding and subsequent packaging of multiple viral genomes inside the same collective infectious unit permits to deliver multiple viral genomes 
to the same cell simultaneously, with potential implications for viral pathogenesis, antibody escape, and antiviral resistance (Leeks et al., 2019; Cuevas et al., 2020).

Our aim is to investigate whether SARS-Cov-2 particles are able to stick together through virion-virion binding and to form aggregates that could stand for CIU. Clues from the literature support our hypothesis. It is noteworthy that many animal viruses, including SARS-Cov-2, are enveloped in a lipid bilayer taken up from host's cellular membranes: because viral surface proteins bind to these membranes to initiate infection, it has been hypothesized that free virions may also be capable of interacting extracellularly with the envelopes of other virions (Cuevas et al., 2020). Vesicular stomatitis virus (VSV) displays features in common with SARS-Cov-2: both are single RNA viruses and both spread with saliva. It has been demonstrated that free VSV particles can spontaneously aggregate into multi-virion topological clusters, in which sociable particles have proximity to each other in a form of proximal convergence (Di Concilio A, Naimpally SA, 1987; Peters and Guadagni, 2016). This allowing speeding of early infection stages in different cell types and co-transmission of diverse viral genetic variants to the same target cell (Andreu-Moreno and Sanjuán, 2020). A topological cluster is a collection of particles that are close to each other (Leader, 1959). In purified virions, this process is driven by protein-lipid interactions probably involving the VSV surface glycoprotein and phosphatidylserine (Cuevas et al., 2020). It is noteworthy that episodic VSV assemblies spontaneously aggregate in the presence of saliva: this feature could increase viral loads and favour infection spreading from a host to another. This might be valid not just in case of VSV, but also of SARS-Cov-2.

\section{MATERIALS AND METHODS}

We examined free-available microscopic images of SARS-Cov and SARS-COV-2 emerging from the surface of cultured cells. Concerning the SARS-Cov pictures, we assessed transmission electron microscope (TEM) images from Groneberg et al. (2005) and ultra-high resolution scanning electron microscopies of the three-dimensional features of SARS-Cov purified from the culture supernatant of Vero E6 cells (Lin et al., 2004).

Concerning the SARS-Cov-2 pictures, we assessed TEM images of virions emerging from the surface of lab cultured cells produced by NIAID's Rocky Mountain Laboratories in Hamilton, Montana: https://www.flickr.com/photos/niaid/albums/72157712914621487.

Furthermore, we examined the movie made available by the Peter Doherty Institute for Infection and Immunity. Indeed, their scientists have successfully grown SARS-Cov-2 from patient samples: https://www.youtube.com/watch?v=qTt3P5V8M1A\&feature=youtu.be.

\section{RESULTS}

We evalauted the microscopic appearance of SARS-Cov and SARS-Cov-2 at different magnifications. Figure 1 depicts available images of SARS-Cov and SARS-Cov-2 particles. A large number of virus particles were found either isolated or in aggregates, where single virions tend to squeeze. The description provided by Lin et al. (2004) for SARSCov particles fully matches the findings detectable in SARS-Cov-2 images: some of the round virus particles of both the coronaviruses tightly adhere with their projections sticking into each other, forming a mosaic patch that leads virions to squeeze and lose their spherical shape. Figure $\mathbf{2 A}$ illustrates the temporal progression of SARS-Cov-2 particles growth in supernatant: note how, with time passing, virions tend to aggregate in small clusters that progressively tend to fill the whole available liquid space with net-like propagations. These propagations are easily detectable in the video available at: https://www.youtube.com/watch?v=qTt3P5V8M1A\&feature=youtu.be. Figure 2B shows collective aggregates of SARS-Cov-2 particles emerging from surfaces of cultured cells. When the virions exit the host cells, single particles tend at first to aggregate in spherical assemblies, then to develop interconnected network-like branches and nodes. 

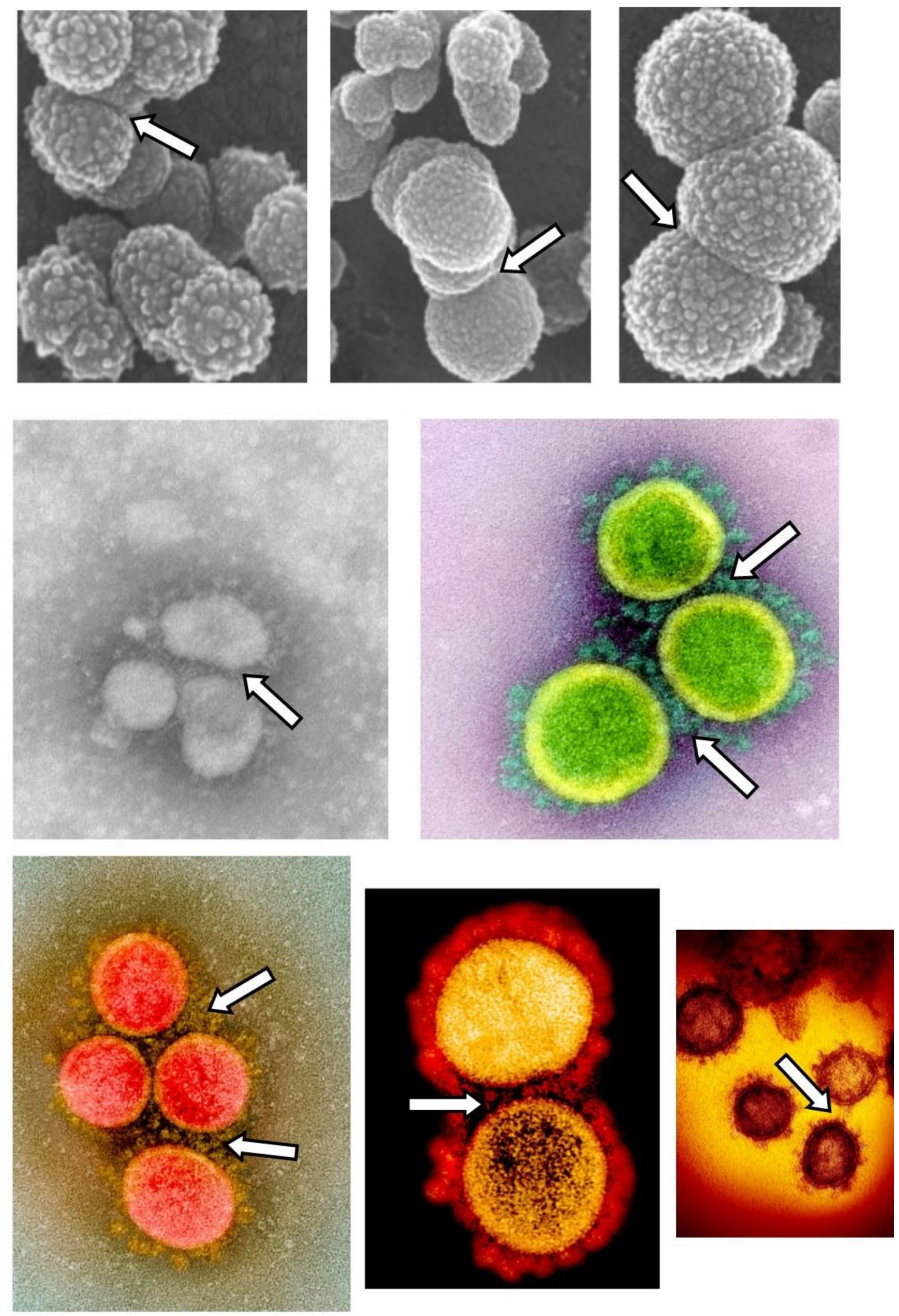

Figure 1. Interaction of coronavirus particles at different magnifications. The black and white pictures illustrate the appearance of SARS-Cov particles and their interface. Modified from Lin et al. (2004) and Groneberg et al. (2005). The coloured pictures illustrate the appearance of SARS-Cov-2 particles and their interface. The virions isolated from a patient emerge from the surface of lab cultured cells. Modified from: https://www.flickr.com/photos/niaid/49534865371/in/photostream/. The arrows point to the adhesion surfaces between different virions. 

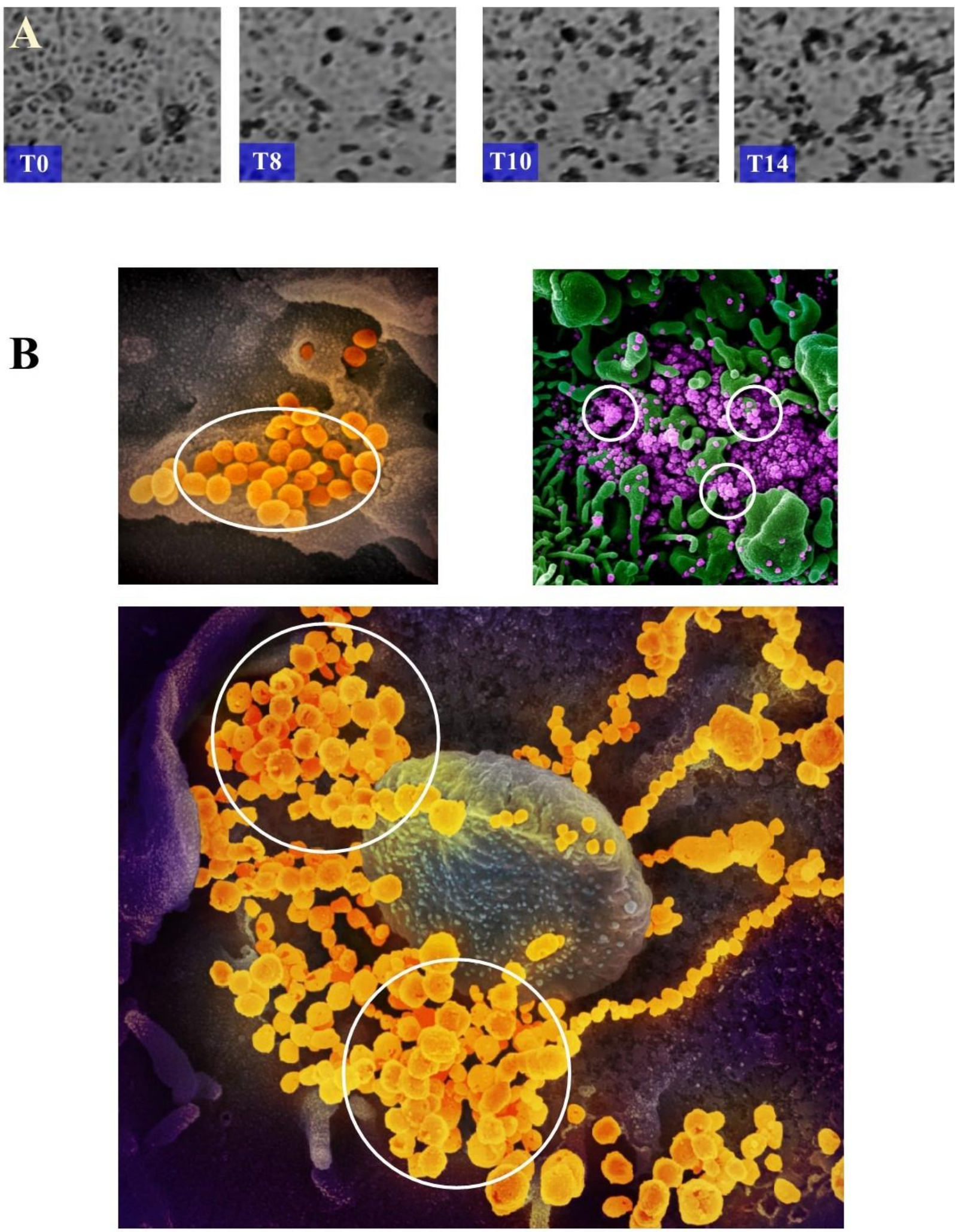

Figure 2. Collective arrangements of multiple SARS-Cov-2 particles. Figure 2A: movie frames illustrating the temporal outcomes of virion production and growth in the supernatant of infected cultured cells. Modified from: https://www.youtube.com/watch?v=qTt3P5V8M1A\&feature=youtu.be. Note the progressive crowding of virion particles, that congregate in small clusters converging in bigger ones with time passing. Figure 2B: scanning electron microscope images showing SARS-CoV-2 virions emerging from the surface of cultured infected cells. Note how small clusters tend to converge and give rise to larger net-like aggregates of numerous particles. Modified from: https://www.flickr.com/photos/niaid/albums/72157712914621487. 


\section{CONCLUSIONS}

Examining available microscopic images of coronaviruses, we found clues pointing towards the occurrence of (possibly transient) SARS-Cov-2 aggregates, in which virions tend to be amassed in multiparticular complexes. Collective spread of viruses may occur through widely different mechanisms, including virion aggregation driven by specific extracellular components, cloaking inside lipid vesicles, encasement in protein matrices, extracellular viral assemblies that resemble bacterial biofilms, virion binding to microbes and dendritic cells (Sanjuán 2017; Segredo-Otero and Sanjuán, 2019). Concerning SARS-Cov-2, the assessed images point towards a peculiar mechanism of particles aggregation: virionvirion interaction, which leads to the formation of progressively growing globular viral assemblies. We hypothesize that such aggregates might contribute to COVID-19 spread rates and SARS-Cov-2's cell-to-cell spread. It is well known that individual virions typically fail to infect cells (Sanjuán, 2018). The causes/implications of low single-virion infectivity are unclear. In principle, this could be due to intrinsic genetic and/or structural defects, but also to host infection barriers that limit early viral proliferation (Sanjuán, 2018). Nevertheless, viral particles' collective dispersal may impact the outcome of the infection, independent of transmission bottlenecks. In touch with this hypothesis, Andreu-Moreno and Sanjuán (2020) found that saliva-driven aggregation has a dual effect on VSV fitness: whereas aggregation tends to increase infectivity in the short term, virion assemblies are highly susceptible to invasion by noncooperative defective variants after a few viral generations.

The ability to build particles assemblies and achieve collective dynamical behavior may provide invaluable advantages to SARS-Cov-2. To provide an example, non-overlapping spheres within a three-dimensional Euclidean space are subjected to constrained arrangements. This leads to the geometrical issue of the sphere packing problem, that aims to find arrangements in which spheres fill as much of the space as possible (Fu et al., 2016; Teich et al., 2016). When SARS-Cov and SARS-Cov-2 particles stick into each other, they form a mosaic patch that squeezes the single virions. This loss of spherical shape modifies sphere packing in three dimensions, because an increased number of viral particles is able to fill up a given amount of available space. By achieving a best package, a growth in local density of crowded viral particles in host's liquid is achieved, maximizing viral load. The enlarged number of virions may lead also to an increase in viscosity of the infected sputum and/or other biological fluids, providing protection against host removal or environmental noxae. SARS-CoV-2 positive patients with few/no symptoms and modest levels of detectable viral RNA in the oropharynx have been described (Zou et al., 2020). This finding, together with the observation that SARS-CoV-2 displays a well-known decay rate both in aerosols and various surfaces (van Doremalen et al., 2020), suggests an intriguing possibility: reduced viral loads could be correlated with decreased viral ability to build particles clustering. When otherwise healthy subjects are exposed to very low viral loads or disassembled CIU, a partial immunization might occur that prevents severe COVID-19 forms. This mechanism, that we might term "mithridatization", has been described in animal models of the 2009 pandemic influenza virus, where decreased challenge dose resulted in reduction in clinical signs and delay in virus production in the upper respiratory tract (Marriott et al., 2014; Peiris et al., 2003).

The globular-like arrangement of multiple SARS-CoV-2 virions may provide another advantage against host immunity and environmental harms: even if immune systems or environmental factors engage the external core of the viral assembly, an inner viral sanctuary might be spared from further damages. It is noteworthy that, while VSV multi-virion complexes occur unfrequently in standard cell cultures, they are abundant in other fluids such as saliva (Cuevas et al., 2020). This suggests that the several coronaviruses ensembles detectable in cellular cultures could be multiplied in human host's fluids such as saliva, sputum, and bronchoalveolar lavage.

A further suggestion that might contribute to solve an intriguing conundrum can be provided: during the current COVID-19 outbreak, pediatric population is affected by less severe symptoms and is rarely admitted to hospital care (Huang et al., 2020; Xu et al., 2020), despite infection transmission displays the same rates and features in children and adults (Bi et al., 2020). Many tentative explanations have been provided for this puzzling phenomenon, including, e.g., the possibility of cross-reaction between SARS-CoV-2 and childhood vaccines (Tozzi and D'Amato, 2020). Here we speculate that the lower symptomatic response in children could be correlated with local factors endowed in the pediatric respiratory airways that are able to scatter the viral assemblies responsible for symptoms severity.

In sum, our results point towards the possibility of clustered SARS-Cov-2 dissemination. Such assemblies might stand for an unnoticed mechanical device that by one side contributes to viral spread, from another side stands for a potential target leading to novel antiviral strategies. 


\section{REFERENCES}

1) Andreu-Moreno I, Sanjuán R. 2020. Collective Viral Spread Mediated by Virion Aggregates Promotes the Evolution of Defective Interfering Particles. mBio, 11(1). pii: e02156-19. doi: 10.1128/mBio.02156-19.

2) Bi Q, Wu Y, Mei S, Ye C, Zou W, et al. 2020. Epidemiology and Transmission of COVID-19 in Shenzhen China: Analysis of 391 cases and 1,286 of their close contacts. medRxiv, doi: https://doi.org/10.1101/2020.03.03.20028423

3) Bou JV, Geller R, Sanjuán R. 2019. Membrane-Associated Enteroviruses Undergo Intercellular Transmission as Pools of Sibling Viral Genomes. Cell Rep. 2019 Oct 15;29(3):714-723.e4. doi: 10.1016/j.celrep.2019.09.014.

4) Cuevas JM, Durán-Moreno M, Sanjuán R. 2017. Multi-virion infectious units arise from free viral particles in an enveloped virus. Nat Microbiol, 2:17078. doi: 10.1038/nmicrobiol.2017.78.

5) Di Concilio A, Naimpally SA. 1987. Proximal convergence, Monatsh. Mathematik vol. 103, no. 2, 93-102, MR0881715.

6) Fu L, Steinhardt W, Zhao H, Socolar JE, Charbonneau P. 2016. Hard sphere packings within cylinders. Soft Matter., 12(9):2505-14. doi: 10.1039/c5sm02875b.

7) Groneberg DA, Hilgenfeld R, Zabel P. 2005. Molecular mechanisms of severe acute respiratory syndrome (SARS). Respiratory Research,6(8).

8) Huang C, Wang Y, Li X, Ren L, Zhao J, Hu Y, et al. 2020. Clinical features of patients infected with 2019 novel coronavirus in Wuhan, China. Lancet, 395, 10223: 497-506.

9) Leader S. 1959. On clusters in proximity spaces. Fundamenta Mathematica 47, 205-213.

10) Leeks A, Sanjuán R, West SA. 2019. The evolution of collective infectious units in viruses. Virus Res, 265:94-101. doi: 10.1016/j.virusres.2019.03.013.

11) Lin Y, Yan X, Cao W, Wang C, Feng J, et al. Probing the structure of the SARS coronavirus using scanning electron microscopy. AntivirTher. 2004 Apr;9(2):287-9.

12) Marriott AC, Dove BK, Whittaker CJ, Bruce C, Ryan KA, et al. 2014. Low dose influenza virus challenge in the ferret leads to increased virus shedding and greater sensitivity to oseltamivir. PLoS One, 9(4):e94090. doi: 10.1371/journal.pone.0094090.

13) Millen S, Gross C, Donhauser N, Mann MC, Péloponèse JM Jr, Thoma-Kress AK. 2019. Collagen IV (COL4A1, COL4A2), a Component of the Viral Biofilm, Is Induced by the HTLV-1 Oncoprotein Tax and Impacts Virus Transmission. Front Microbiol, 10:2439. doi: 10.3389/fmicb.2019.02439.

14) Pais-Correia A-M, Sachse M, Guadagnini S, Robbiati V, Lasserre R, et al. 2010. Biofilm-like extracellular viral assemblies mediate HTLV-1 cell-to-cell transmission at virological synapses. Nature Medicine, 16:8389.

15) Peiris JS, Chu CM, Cheng VC, Chan KS, Hung IF, et al. 2003. Clinical progression and viral load in a community outbreak of coronavirus-associated SARS pneumonia: a prospective study. Lancet. 2003 May 24;361(9371):1767-72.

16) Peters JF, Guadagni C. 2016. Strongly proximal continuity \& strong connectedness. Topololgy and its Applications. Vol 204, 41-50, doi: 10.1016/j.topol.2016.02.008.

17) Sanjuán R. 2017. Collective Infectious Units in Viruses. Trends Microbiol, 25(5):402-412. doi: 10.1016/j.tim.2017.02.003.

18) Sanjuán R. 2018. Collective properties of viral infectivity. CurrOpinVirol, 1-6. doi: 10.1016/j.coviro.2018.06.001.

19) Sanjuán R1, Thoulouze MI. 2019. Why viruses sometimes disperse in groups? Virus Evol, 5(1):vez014. doi: 10.1093/ve/vez014.

20) Segredo-Otero E, Sanjuán R. 2019. The effect of genetic complementation on the fitness and diversity of viruses spreading as collective infectious units. Virus Res., 267:41-48. doi: 10.1016/j.virusres.2019.05.005.

21) Teich EG, van Anders G, Klotsa D, Dshemuchadse J, Glotzer SC. 2016. Clusters of polyhedra in spherical confinement. Proc Natl Acad Sci U S A., 113(6):E669-78. doi: 10.1073/pnas.1524875113.

22) Thoulouze MI, Alcover A. 2011. Can viruses form biofilms? Trends Microbiol, 19(6):257-62.doi: 10.1016/j.tim.2011.03.002.

23) Tozzi A, D'Amato G. 2020. Cross-reactivity between COVID-19 and childhood vaccines? Electronic response to:del Rio C; Malani PN. 2020. 2019 Novel Coronavirus-Important Information for Clinicians. JAMA. Published online February 5, 2020. doi:10.1001/jama.2020.1490.

24) van Doremalen N, Bushmaker T, Morris DH, Holbrook MG, Gamble A, et al. 2020. Aerosol and Surface Stability of SARS-CoV-2 as Compared with SARS-CoV-1. NEJM, DOI: 10.1056/NEJMc2004973

25) Vidakovic L, Singh PK, Hartmann R, Nadell CD, Drescher K. 2018. Dynamic biofilm architecture confers individual and collective mechanisms of viral protection. Nature Microbiology, 3:26-31.

26) Zou L, Ruan F, Huang M, Liang L, Huang H, et al. 2020. SARS-CoV-2 Viral Load in Upper Respiratory Specimens of Infected Patients. N Engl J Med 2020; 382:1177-1179. DOI: 10.1056/NEJMc2001737. 